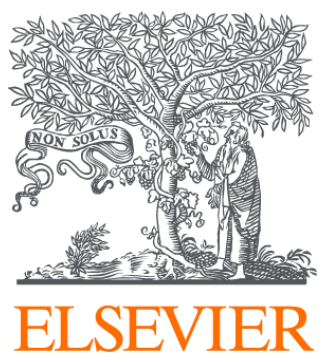

Since January 2020 Elsevier has created a COVID-19 resource centre with free information in English and Mandarin on the novel coronavirus COVID-

19. The COVID-19 resource centre is hosted on Elsevier Connect, the company's public news and information website.

Elsevier hereby grants permission to make all its COVID-19-related research that is available on the COVID-19 resource centre - including this research content - immediately available in PubMed Central and other publicly funded repositories, such as the WHO COVID database with rights for unrestricted research re-use and analyses in any form or by any means with acknowledgement of the original source. These permissions are granted for free by Elsevier for as long as the COVID-19 resource centre remains active. 


\title{
Extent of pulmonary thromboembolic disease in patients with COVID-19 on CT: relationship with pulmonary parenchymal disease
}

\author{
C. Fang ${ }^{\text {a }}$, G. Garzillo ${ }^{\mathrm{a}, *}$, B. Batohi ${ }^{\mathrm{a}}$, J.T.H. Teo ${ }^{\mathrm{b}}$, M. Berovic ${ }^{\mathrm{a}}$, P.S. Sidhu ${ }^{\mathrm{a}}$, \\ H. Robbie ${ }^{a}$
}

a Department of Radiology, King's College Hospital NHS Foundation Trust, Denmark Hill, Brixton, London SE5 9RS, UK

${ }^{\mathrm{b}}$ Department of Neurology, King's College Hospital NHS Foundation Trust, Denmark Hill, Brixton, London SE5 9RS, UK

\section{ARTICLE INFORMATION}

\section{Article history:}

Received 9 June 2020

Accepted 7 July 2020
AIM: To report the severity and extent of pulmonary thromboembolic disease (PTD) in COVID-19 patients undergoing computed tomography pulmonary angiography (CTPA) in a tertiary centre.

MATERIALS AND METHODS: This is a retrospective analysis of COVID-19 patients undergoing CTPA over a period of 27 days. The presence, extent, and severity of PTD were documented. Two observers scored the pattern and extent of lung parenchymal disease including potential fibrotic features, as well as lymph node enlargement and pleural effusions. Consensus was achieved via a third observer. Interobserver agreement was assessed using kappa statistics. Student's t-test, chi-squared, and Mann-Whitney $U$-tests were used to compare imaging features between PTD and non-PTD sub-groups.

RESULTS: During the study period, 100 patients with confirmed COVID-19 underwent CTPA imaging. Ninety-three studies were analysed, excluding indeterminate CTPA examinations. Overall incidence of PTD was 41/93 (44\%) with 28/93 patients showing small vessel PTD (30\%). D-dimer was elevated in 90/93 (96.8\%) cases. A high Wells' score did not differentiate between PTD and non-PTD groups ( $p=0.801)$. The interobserver agreement was fair (kappa=0.659) for parenchymal patterns and excellent (kappa=0.816) for severity. Thirty-four of the 93 cases (36.6\%) had lymph node enlargement; $29 / 34$ (85.3\%) showed no additional source of infection. Sixteen of the 93 (17.2\%) cases had potential fibrotic features.

CONCLUSION: There is a high incidence of PTD in COVID-19 patients undergoing CTPA and lack of a risk stratification tool. The present data indicates a higher suspicion of PTD is needed in severe COVID-19 patients. The concomitant presence of possible fibrotic features on CT indicates the need for follow-up.

Crown Copyright (c) 2020 Published by Elsevier Ltd on behalf of The Royal College of Radiologists. All rights reserved.

\footnotetext{
* Guarantor and correspondent: G. Garzillo, King's College Hospital NHS Foundation Trust, Denmark Hill, Brixton, London SE5 9RS, UK. Tel.: +07969772639. E-mail address: giorgio.garzillo@nhs.net (G. Garzillo).
} 


\section{Introduction}

Coronavirus disease 2019 (COVID-19), the pandemic caused by the novel coronavirus known as severe acute respiratory syndrome coronavirus 2 (SARS-CoV-2), was first identified in Wuhan, China, in December 2019. ${ }^{1}$ Since then, the virus has spread rapidly worldwide and as of 8 May 2020 , there are over 3.7 million reported cases of COVID-19 globally with over 260,000 deaths. ${ }^{2}$ The majority of patients with COVID-19 present with an acute respiratory illness of varying severity with fever, cough, and dyspnoea being the most common symptoms.,

The diagnosis of COVID-19 is usually confirmed by identification of viral RNA using reverse-transcription polymerase chain reaction (RT-PCR); however, the RT-PCR test is subject to variability depending on sampling technique with positive detection rate ranging between $30-60 \%{ }^{5}$ Hence, imaging has become an important tool in the diagnosis and management of patients with COVID-19, initially prompted by findings of a study by Ai et al. ${ }^{6}$ confirming high sensitivity of computed tomography (CT) in diagnosing COVID-19; however, the use of CT as a first-line imaging technique remains controversial and in most radiology departments, CT is reserved for cases with severe disease or those with stagnating or deteriorating clinical conditions. ${ }^{7-9}$ In the wake of the pandemic, various radiological societies and colleges have set guidance on how to interpret the pattern and extent of radiographic and CT findings in patients with suspected or confirmed COVID$19^{10-13}$; however, none of the existing guidance has been externally validated in larger cohorts. In addition, despite several reports investigating the relationship between poor outcomes and various clinical and biochemical factors, ${ }^{3,4,14,15}$ the focus on radiological parameters remains somewhat limited.

Coagulopathy is emerging as one of the common complications of COVID-19 16,17 and several reports have concluded that high D-dimer values, prolonged prothrombin time, and thrombocytopenia are associated with poor outcome. $^{3,15,18-22}$ The pathophysiology is not well understood, but emerging evidence points towards hyperinflammation resulting in a vascular disease within the lungs that is primarily characterised by thrombotic microangiopathy. ${ }^{22,23}$ Recently published data in a small cohort of hospitalised patients with severe COVID-19 suggest up to $40 \%$ incidence of pulmonary thromboembolic disease (PTD). ${ }^{24,25}$ The recent report from the National Institute for Public Health of the Netherlands concluded that the thrombotic state can occur in a substantial percentage of COVID-19 patients, but highlighted the paucity of data on the prevalence of PTD. ${ }^{26}$

The aim of the study was to report the severity and extent of PTD in patients with confirmed COVID-19 who have undergone CT pulmonary angiography (CTPA) imaging in a tertiary centre. The secondary aims are 1 . to assess the effectiveness of the British Society of Thoracic Imaging (BSTI) current guidance on the pattern and extent of lung parenchymal abnormalities in COVID-19, and 2. to assess whether there is any difference in the patterns and severity of pulmonary parenchymal disease on CT in COVID-19 patients with and without PTD.

\section{Material and methods}

\section{Study population}

A single-centre retrospective analysis of all consecutive patients with confirmed SARS-CoV-2 on RT-PCR who underwent a CT CTPA study between 23 March 2020 and 19 April 2020 was performed. This project was granted approval by the institutional Ethics committee. Patient consent was not required due to retrospective nature of the study.

\section{CTPA protocol}

All CT examinations were acquired using a GE Discovery CT750 HD (64 section) CT machine (GE Healthcare, Milwaukee, Wisconsin, USA) using the departmental COVID-19 CTPA protocol for PTD $(1.25 \mathrm{~mm}$ section thickness, peak tube voltage of $100 \mathrm{kVp}$, and current modulation range between $80-500 \mathrm{mAs}$, tube rotation time of 0.5 seconds, and a pitch of 0.984:1) which includes a volumetric non-contrast high-resolution CT thorax prior to intravenous administration of $60 \mathrm{ml}$ non-ionic contrast medium with $100 \mathrm{ml}$ saline chaser at a rate of $4.5 \mathrm{ml} / \mathrm{s}$ with a time delay of 6 seconds, using a bolus-tracking technique, set at the pulmonary phase. All patients were scanned in a supine position from lung apices to bases at full inspiration. All images were reconstructed using a high-spatial-frequency soft kernel (WW/WL 400/40, $0.625 \mathrm{~mm}$ sections). Contiguous images of 1-mm thickness were reviewed on lung window settings for parenchymal disease (width, 1,500 $\mathrm{HU}$; level, $-500 \mathrm{HU}$ ) and at mediastinal window settings for PTD and lymph node enlargement (width, $350 \mathrm{HU}$; level, $50 \mathrm{HU}$ ). The unenhanced thoracic CT was performed to accurately assess subtle parenchymal abnormalities, such as ground-glass infiltrates, that can be masked by contrast medium.

\section{Data collection}

Patient demographic, clinical, laboratory, and outcome data were extracted from electronic medical records. Laboratory data collected were from the date closest to the CTPA study date. Duration of clinical symptoms was defined from the self-reported onset of COVID-19 symptoms to the date of CTPA. Wells' scores for PTD were calculated. ${ }^{27}$ Outcome data were updated until 3 May 2020, which was 2 weeks after the end of study inclusion period.

\section{Image analysis}

All CT studies were reviewed by two experienced radiologists (with 4 years of experience in cancer imaging and with 6 years of experience in thoracic imaging, respectively). CT patterns and severity of lung parenchymal disease, severity and extent of PTD, presence of CT signs of 
Table 1

Various computed tomography (CT) patterns that could represent, raise suspicion of, or suggest alternative diagnosis for COVID-19 pneumonia as per British Society of Thoracic Imaging (BSTI) guidance.

\begin{tabular}{|c|c|}
\hline CT patterns & CT findings \\
\hline \multirow{3}{*}{$\begin{array}{l}\text { Classic } \\
\text { COVID-19 pneumonia }\end{array}$} & Peripheral GGO \\
\hline & Possible crazy-paving pattern ${ }^{a}$ \\
\hline & Organising pneumonia ${ }^{\mathrm{b}}$ \\
\hline \multirow{4}{*}{$\begin{array}{l}\text { Probable } \\
\text { COVID-19 pneumonia }\end{array}$} & Mixture of bronchocentric and peripheral \\
\hline & $\begin{array}{l}\text { consolidation predominantly in the lower } \\
\text { zones }\end{array}$ \\
\hline & Organising pneumonia \\
\hline & GGO scarce \\
\hline \multirow[t]{2}{*}{ Indeterminate } & $\begin{array}{l}\text { Does not fit in the three other categories } \\
\text { with diffuse/patchy GGO that are not } \\
\text { peripheral, fibrosis with ground glass, } \\
\text { complex CT patterns }\end{array}$ \\
\hline & $\begin{array}{l}\text { Similar patterns to the above but clinical } \\
\text { context is wrong or suggestive of } \\
\text { alternative diagnosis }\end{array}$ \\
\hline \multirow[t]{3}{*}{ Non-COVID-19 } & Lobar pneumonia \\
\hline & Cavitating consolidation \\
\hline & $\begin{array}{l}\text { Tree-in-bud/centrilobular opacities, lymph } \\
\text { node enlargement, pleural effusions }\end{array}$ \\
\hline
\end{tabular}

GGO, ground-glass opacity.

a Crazy paving pattern on CT includes GGO with inter/intralobular septal thickening.

b Organising pneumonia patterns on CT are bronchocentric/peripheral consolidation with reversed halo sign and peri-lobular pattern. ${ }^{25}$

pulmonary hypertension, CT features of possible fibrosis, and intrathoracic lymph node enlargement were reviewed. A consensus was reached by a third radiologist (11 years of experience in thoracic imaging) where there was a disagreement. All readers were blinded to patient clinical details and existing radiology reports. CT pattern and the extent of parenchymal disease was documented as normal, classic, probable, indeterminate or non-COVID-19, and mild, moderate, and severe, respectively, as per BSTI guidelines (Table 1 ). The CT severity was defined as mild if up to three focal abnormalities of $3 \mathrm{~cm}$ in maximum diameter. Severity of PTD was classified as subsegmental, segmental, lobar, main, and saddle. The extent of PTD was documented based on the number of pulmonary lobes involved (between 1-6); with lingula considered a separate lobe. Potential features of fibrosis were considered present if two of the following were observed: traction bronchiectasis away from the areas of consolidation but within the areas of ground-glass opacification, volume loss as judged by position of the oblique fissures, ${ }^{28}$ and architectural distortion as per Fleischner Society glossary of terms. $^{29}$ The patterns of potential fibrosis on CT were categorised into possible fibrotic organising pneumonia or possible fibrosis associated with acute respiratory distress syndrome (ARDS), also based on Fleischner Society glossary of terms. ${ }^{29}$ Intrathoracic lymph nodes were considered enlarged if they measured $>10 \mathrm{~mm}$ or $>3 \mathrm{~mm}$ in short axis diameter for mediastinal and hilar lymph nodes, respectively. ${ }^{30} \mathrm{CT}$ signs of pulmonary hypertension was

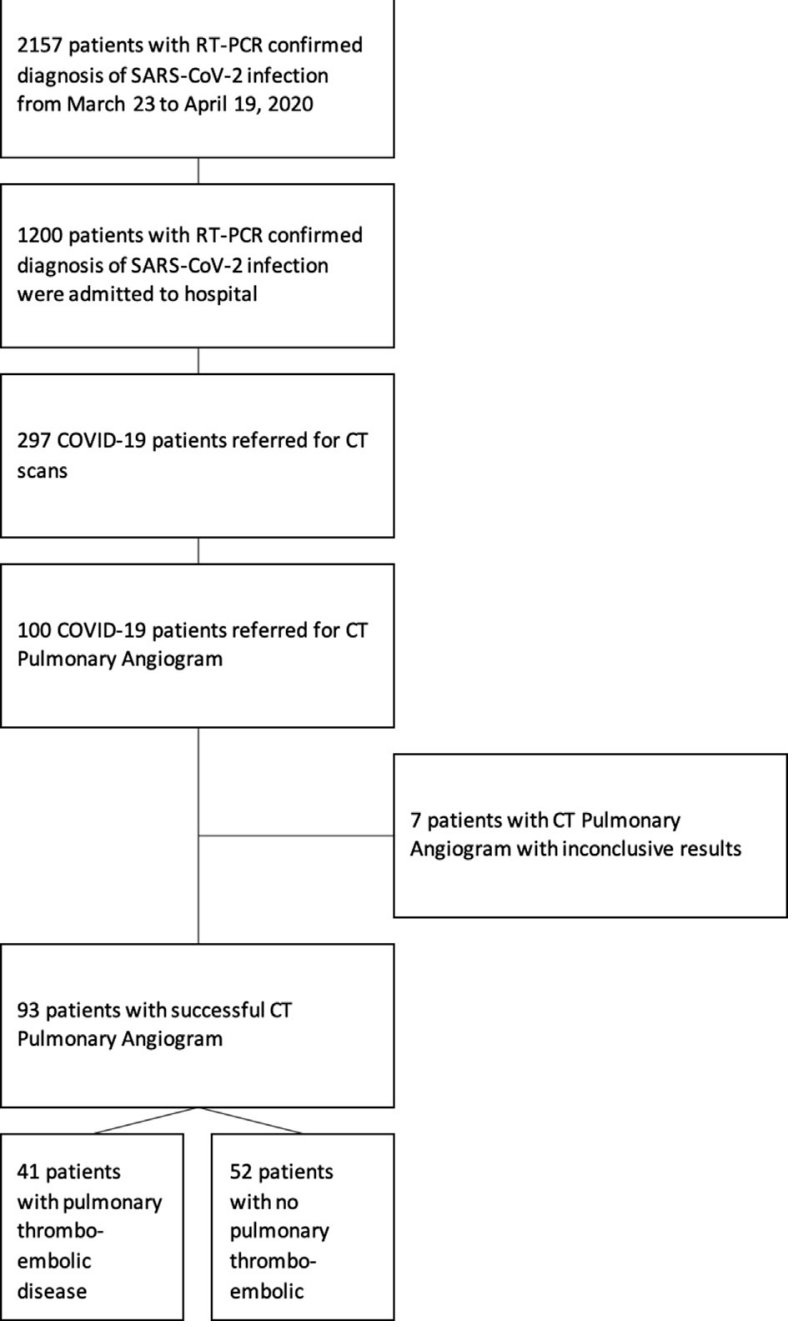

Figure 1 Consort diagram illustrating the process of selecting patients for the final study population.

defined as pulmonary artery diameter $>30 \mathrm{~mm}$, pulmonary artery diameter to ascending thoracic aorta ratio $>1$, and contrast medium reflux into the inferior vena cava/ hepatic veins. ${ }^{31,32}$

\section{Statistical analysis}

Statistical analysis was performed by using SPSS software (version 23; IBM, Chicago, IL, USA), and $p$-values $<0.05$ were considered to indicate statistical significance. The normality of data was assessed using the Shapiro-Wilk test. Continuous variables were reported as mean \pm standard deviation or median (interquartile range) as appropriate. Kappa statistics were used to assess interobserver agreement with the level of agreement classified as: poor (kappa $\leq 0.40)$, fair $(0.4<\operatorname{kappa} \leq 0.6)$, good $(0.6<\mathrm{kappa} \leq 0.75)$, excellent $(0.75<$ kappa $<1.0){ }^{33}$ Student's $t$-test, chi-squared test, Mann-Whitney $U$-test where appropriate were used 
to compare study parameters between the groups based on the normality of the data.

\section{Results}

Patent demographics, clinical, laboratory data within PTD and non- PTD subgroups

There were 2,157 patients with positive RT-PCR tests for SARS-CoV-2 during the study period (Fig 1). Out of 297 patients referred for CT imaging, 100 CTPA studies were performed in 97 COVID-19 patients. Seven studies were excluded from final analysis due to non-diagnostic quality. CTPAs were referred from the emergency department $(n=24 / 93)$, inpatient wards $(n=49 / 93)$, and intensive care units $(n=20 / 93)$. Patient demographic, clinical and laboratory, and outcome data are listed in Table 2. D-dimer was elevated in 90/93 (96.8\%) of study cases. The median duration of symptoms to CTPA studies in patients referred from the emergency department, inpatient wards, intensive care unit was 8.5, 16, and 21 days, respectively, and the overall median duration of symptoms to CTPA studies was 14 days. The overall incidence of PTD was $44 \%$ ( $n=41 / 93)$. In

Table 2

Demographic, clinical and laboratory findings from the patients at the time of computed tomography pulmonary angiography (CTPA) study.

\begin{tabular}{|c|c|c|c|}
\hline & \multicolumn{2}{|c|}{ Presence of pulmonary thromboembolic disease } & \multirow{2}{*}{$\begin{array}{l}\text { Unadjusted } \\
p \text {-value }\end{array}$} \\
\hline & Yes & No & \\
\hline Number of patients & 41 & 52 & \\
\hline \multicolumn{4}{|l|}{ Parameter } \\
\hline Age, median (IQR) & $62(56-69)$ & $57(50.5-68.5)$ & 0.044 \\
\hline Gender, $n$ & 41 & 52 & 0.499 \\
\hline Female & 13 & 20 & \\
\hline Male & 28 & 32 & \\
\hline Current smoker, $n$ & 5 & 8 & 0.660 \\
\hline \multicolumn{4}{|l|}{ Comorbidity, $n$} \\
\hline Hypertension & 20 & 24 & \\
\hline Diabetes mellitus & 14 & 24 & \\
\hline Chronic obstructive lung disease & 2 & 4 & \\
\hline Malignancy & 6 & 4 & \\
\hline Chronic kidney disease & 6 & 4 & \\
\hline Coronary heart disease & 2 & 7 & \\
\hline Asthma & 2 & 9 & \\
\hline Obesity (BMI > 30) & 13 & 18 & \\
\hline None of the above & 7 & 9 & \\
\hline \multicolumn{4}{|l|}{ Referral source, $n(\%)$} \\
\hline Emergency department & $12(29.3 \%)$ & $12(23.0 \%)$ & \\
\hline Intensive care unit & $13(31.7 \%)$ & $7(13.5 \%)$ & \\
\hline Inpatient ward & $16(39.0 \%)$ & $33(63.5 \%)$ & \\
\hline $\begin{array}{l}\text { COVID-19 symptomatic disease } \\
\text { duration prior to CTPA, days (IQR) }\end{array}$ & $16(9-23)$ & $13(7-17.25)$ & 0.246 \\
\hline Wells scores, $n$ & & & 0.801 \\
\hline$<4$ points & 20 & 24 & \\
\hline$>4$ points & 21 & 28 & \\
\hline \multicolumn{4}{|l|}{ Laboratory findings, median (IQR) } \\
\hline White blood cell count $\left(\times 10^{9} / 1\right)$ & $10.36(8.53-13.98)$ & $7.81(5.64-10.76)$ & 0.058 \\
\hline$<4, n(\%)$ & $0(0 \%)$ & $4(7.7 \%)$ & \\
\hline $4-10, n(\%)$ & $19(46.3 \%)$ & $31(59.6 \%)$ & \\
\hline$>10, n(\%)$ & $22(53.7 \%)$ & $17(32.7 \%)$ & \\
\hline Lymphocyte count $\left(\times 10^{9} / 1\right)$ & $1.11(0.73-1.55)$ & $1.07(0.74-1.59)$ & 0.670 \\
\hline$<0.8, n(\%)$ & $13(31.7 \%)$ & $17(32.7 \%)$ & \\
\hline Neutrophils count $\left(\times 10^{9} / 1\right)$ & $8.52(6.48-11.61)$ & $6.07(4.31-9.67)$ & 0.043 \\
\hline Haemoglobin, g/l & $111(84-126)$ & $121(102-134.5)$ & 0.071 \\
\hline Platelet count, $\times 10^{9} / 1$ & $340(245-412)$ & $289(206-421)$ & 0.521 \\
\hline Albumin, g/l & $27(24-33)$ & $33(29.5-37)$ & 0.001 \\
\hline ALT, U/1 & $44(32-62.5)$ & $45(27.5-80.5)$ & 0.222 \\
\hline Creatinine, $\mu \mathrm{mol} / \mathrm{l}$ & $82(69-142)$ & $73(59.5-126)$ & 0.557 \\
\hline Urea & $9.2(4.8-14.1)$ & $6.7(4.2-9.15)$ & 0.018 \\
\hline C-reactive protein & $146(92-216.3)$ & $73(59.5-126)$ & 0.068 \\
\hline $\mathrm{LDH}, \mu / 1$ & $466(389-600.75)$ & $561.5(439.75-709.25)$ & 0.221 \\
\hline Troponin I & $37(19.5-99)$ & $17(8-34)$ & 0.759 \\
\hline D-dimer & $7465(3835-8000)$ & $2450(1170-3985)$ & 0.001 \\
\hline Serum ferritin, $\mu \mathrm{g} / 1$ & $837.5(616.25-1371.5)$ & $1051.5(678.25-2396.5)$ & 0.138 \\
\hline \multicolumn{4}{|l|}{ Outcomes, $n(\%)$} \\
\hline Death & $6(14.6 \%)$ & $5(9.6 \%)$ & 0.457 \\
\hline
\end{tabular}

p-Values were using Student's t-test, chi-squared, and Mann-Whitney $U$-test respectively.

IQR, interquartile range; BMI, body mass index; ALT, alanine transaminase; LDH, lactate dehydrogenase. 
$12 / 24(50 \%)$ referrals from the emergency department, the studies were positive for PTD, 16/49 (32.7\%) from inpatient wards, and 13/20 (65.0\%) from intensive care. In 28/41 (68.3\%) of cases, there were segmental and subsegmental PTD with 26/41 (63.4\%) having three lobes or fewer affected by pulmonary emboli.

\section{Interobserver agreement}

Interobserver agreement for $\mathrm{CT}$ pattern of parenchymal disease was fair (kappa $=0.576, p<0.001$ ). By grouping the classic and probably categories of lung parenchymal abnormalities, the kappa increased from fair to good (kappa: $0.659, p<0.001)$. The interobserver agreement was excellent for severity of lung parenchymal changes (kappa $=0.816, p<0.001$ ) and fair for pulmonary fibrosis (kappa $=0.594, p<0.001)$.

CT pattern of the lung disease, nodal status, presence of pleural effusion, and extent of pulmonary

thromboembolic disease

CT patterns, severity of parenchymal disease, and the extent and severity of PTD are detailed in Table 3. In 40/93 (43\%) studies, the CT pattern was intermediate for COVID-19 (Fig 2). There were 59/93 (63.4\%) studies with severe parenchymal disease with 30/93 (32.2\%) showing moderate disease (Fig 3). In 16/93 (17.2\%) studies, imaging demonstrated at least two CT signs of possible fibrosis. In 13 (81.3\%) of these cases, the specific pattern of potential fibrotic features on CT was in keeping with fibrotic organising pneumonia rather than possible fibrosis related to ARDS (Fig 4).

There were $34 / 93$ (36.6\%) cases with enlarged mediastinal or hilar lymph nodes. The distribution of lymph node enlargement and pleural effusion among different CT patterns is detailed in Table 4. The frequency of lymph node enlargement was observed equally amongst different CT patterns, between 30-42.5\%. Lymph node enlargement was present in 5/16 (31.3\%) cases who had features of fibrosis versus 29/77 (37.7\%) who did not have fibrotic features. Five cases who had lymph node enlargement also had confirmed super-added fungal infection from blood serum with all of these cases showing lymph node enlargement. In the remainder of $29 / 34$ (85.3\%) cases with lymph nodes enlargement, there was no additional source of infection. Twenty of the 93 (21.5\%) studies in the present cohort had pleural effusion.

The presence of pulmonary hypertension was significantly more common among patients with PTD. The level of D-dimer was significantly higher in patients with severe parenchymal disease compared with moderate disease $(p=0.046)$. The lymph node status and presence of pulmonary fibrosis did not differ among the PTD and non-PTD subgroups (Table 4).

\section{Discussion}

The present study has confirmed the high incidence of PTD in COVID-19 patients who underwent CTPAs. In the
Table 3

Computed tomography (CT) patterns in COVID-19 patients who underwent CT pulmonary angiography (CTPA) studies and extent of pulmonary embolism in patients who are found to have positive CTPA studies.

\begin{tabular}{|c|c|c|c|c|}
\hline & \multicolumn{3}{|c|}{$\begin{array}{l}\text { Presence of pulmonary } \\
\text { embolus }\end{array}$} & \multirow{2}{*}{$\begin{array}{l}\text { Chi-squared } \\
\text { unadjusted } \\
p \text {-values }\end{array}$} \\
\hline & Yes & No & Total & \\
\hline \multicolumn{4}{|l|}{ Lymph node enlargement } & 0.082 \\
\hline Yes & 19 & 15 & 34 & \\
\hline No & 22 & 37 & 59 & \\
\hline \multicolumn{4}{|l|}{ Signs of right heart strain } & $<0.001$ \\
\hline Yes & 26 & 14 & 40 & \\
\hline No & 15 & 38 & 53 & \\
\hline \multicolumn{4}{|l|}{ Fibrosis } & 0.601 \\
\hline Yes & 8 & 8 & 16 & \\
\hline No & 33 & 44 & 77 & \\
\hline \multicolumn{5}{|l|}{ CT Pattern } \\
\hline Classic COVID-19 & 6 & 14 & 20 & \\
\hline Probable COVID-19 & 9 & 15 & 24 & \\
\hline Indeterminate COVID-19 & 21 & 19 & 40 & \\
\hline Non- COVID-19 & 5 & 4 & 9 & \\
\hline \multicolumn{5}{|c|}{ Parenchyma disease severity } \\
\hline Normal & 0 & 1 & & \\
\hline Mild & 2 & 1 & & \\
\hline Moderate & 20 & 10 & & \\
\hline Severe & 30 & 29 & & \\
\hline \multicolumn{5}{|l|}{ Severity of PTD } \\
\hline Subsegmental & 12 & - & & \\
\hline Segmental & 16 & - & & \\
\hline Lobar & 8 & - & & \\
\hline Main & 4 & - & & \\
\hline Saddle & 1 & - & & \\
\hline \multicolumn{5}{|l|}{ Extent of PTD } \\
\hline One lobe & 12 & - & & \\
\hline Two lobes & 6 & - & & \\
\hline Three lobes & 8 & - & & \\
\hline Four lobes & 6 & - & & \\
\hline Five lobes & 4 & - & & \\
\hline Six lobes & 5 & - & & \\
\hline
\end{tabular}

PTD, pulmonary thromboembolic disease.

pre-COVID-19 period, the detection rate of PTD was $4.2 \%$ in the emergency setting without risk stratification and $11.2 \%$ with Wells' score risk stratification, ${ }^{34}$ whereas, in the present COVID-19 cohort, PTD is not only common, but the Wells' score was not discriminatory in estimating the risk of PTD. This is likely due to Well's scoring being weighted towards PTD as the leading diagnosis rather than a secondary diagnosis concurrent with symptomatic lung disease.

Although conventional D-dimer thresholds was nondiscriminatory for PTD (as virtually all COVID-19 patients had elevated D-dimers), higher D-dimer levels were found in COVID-19 patients with PTD versus COVID-19 patients without PTD. This could be explained with higher levels of D-dimer being associated with more severe COVID-19 disease. $^{20}$

The radiological pattern and severity of lung disease does not differ in subgroups of patients with or without PTD. The majority of patients in the present cohort had segmental and sub-segmental PTD with most patients having three or less lobes affected by thromboembolic disease. The present findings are in line with previous studies investigating 


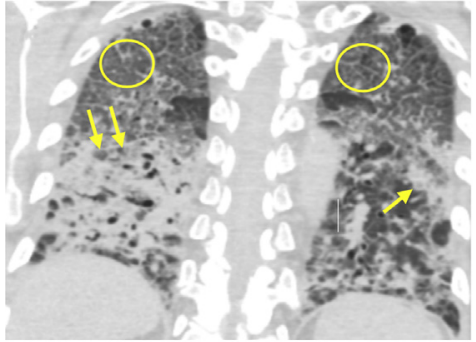

(a)

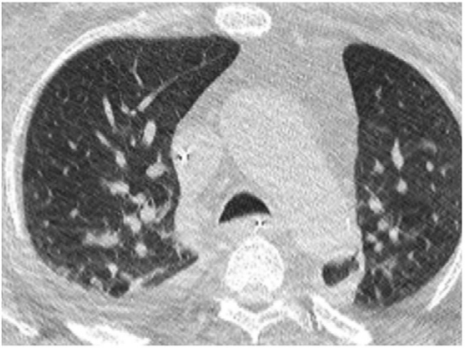

(c)

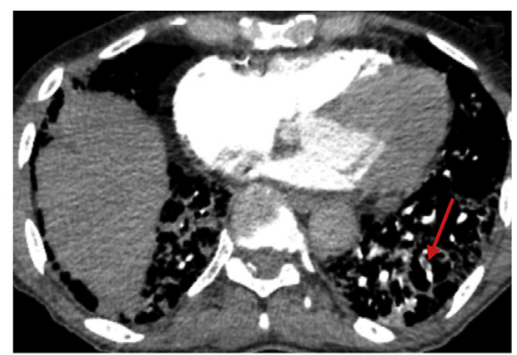

(b)

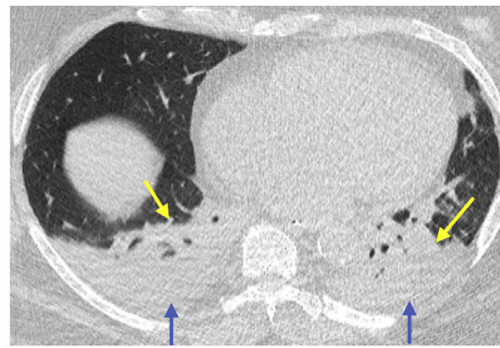

(d)

Figure $2(\mathrm{a}, \mathrm{b})$ Axial and coronal unenhanced CT images of the chest of a 76-year-old man admitted to the ward with COVID-19 and increasing $\mathrm{O}_{2}$ requirements. Note bilateral diffuse crazy paving pattern (yellow circles) and lower-lobe-predominant asymmetrical consolidation (yellow arrows), in keeping with severe disease that is indeterminate for COVID-19. (b) The subsequent axial CTPA image confirming small subsegmental PE in the left lower lobe (red arrow). (c,d) Axial unenhanced CT images of 67-year-old man admitted to intensive care with COVID-19 and failure to respond to treatment. (c) No parenchymal abnormality in the upper lobes with (d) showing bilateral pleural effusions (blue arrows) with lower lobe predominant consolidation and collapse (yellow arrows), in keeping with non-COVID-19 pattern.

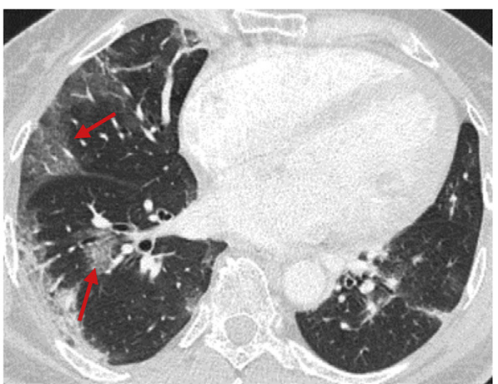

(a)

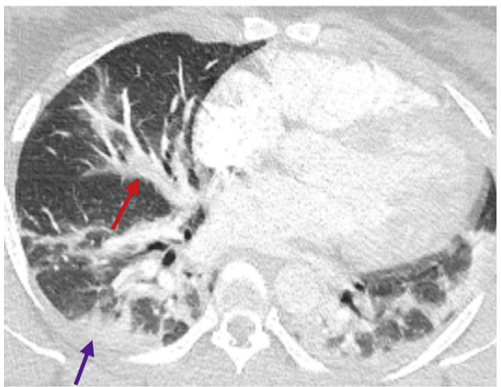

(c)

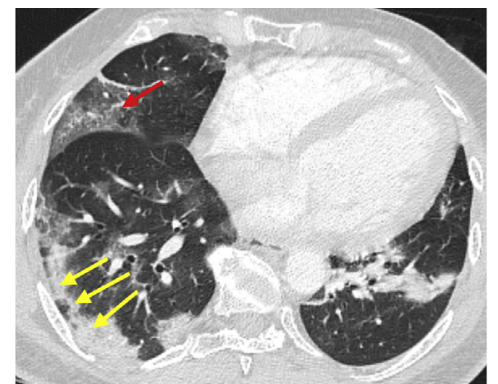

(b)

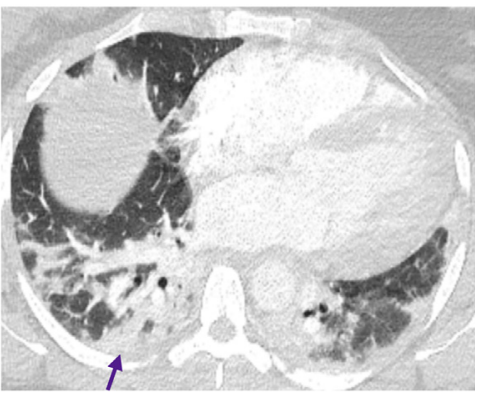

(d)

Figure 3 (a,b) Axial CTPA images of a 36-year-old woman presenting to the emergency department with breathlessness and haemoptysis. Note lower zone predominant peripheral and bronchocentric ground-glass opacification (red arrows) and consolidation with a perilobular pattern (yellow arrows). The pattern is in keeping with moderate classic COVID-19. (c,d) Axial CTPA images of a 37-year-old woman presenting to emergency department with breathlessness. Note bronchocentric (red arrow) and peripheral lower zone consolidation (purple arrows) with no ground-glass opacification. The pattern is in keeping with probable COVID-19, which is moderate in extent. 


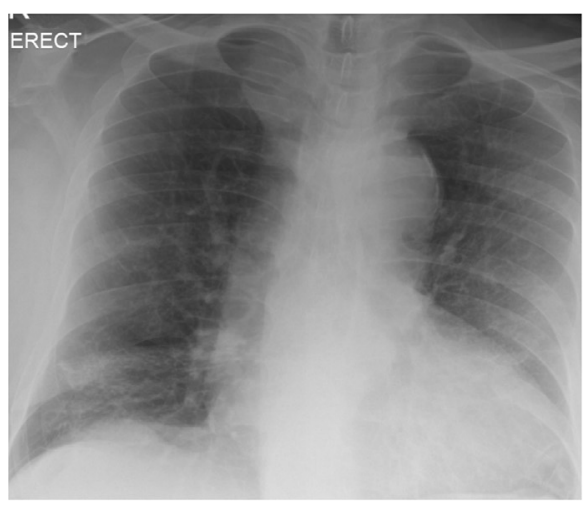

(a)

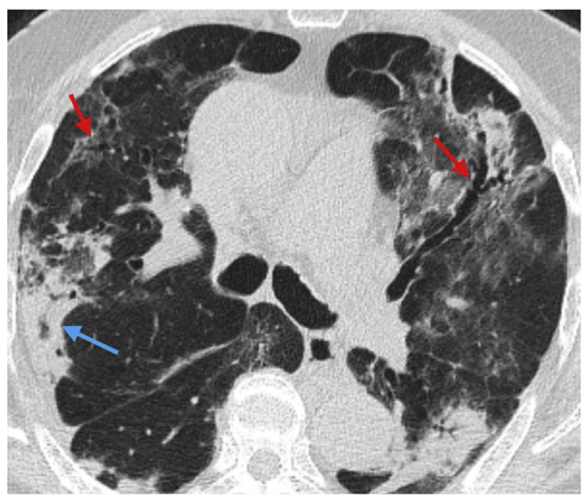

(c)

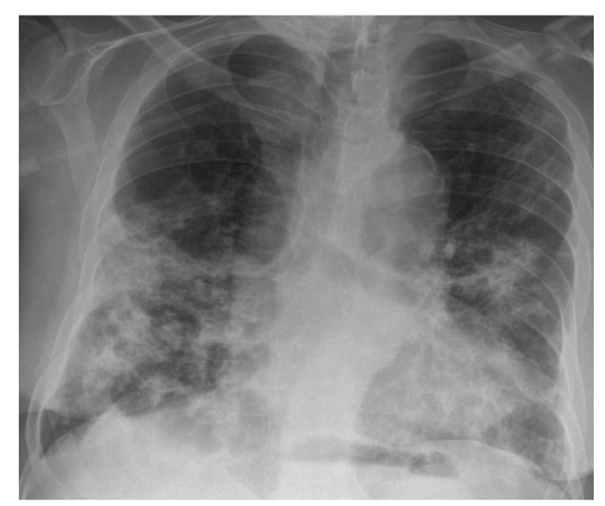

(b)

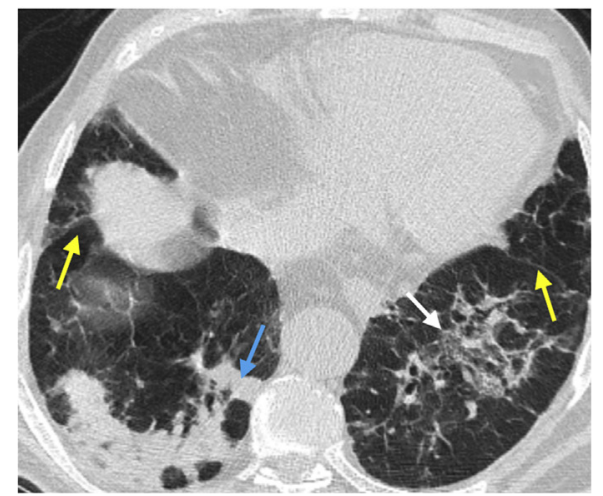

(d)

Figure 4 (a) Baseline chest X-ray and (b) two-week follow-up chest X-ray of an 84-year-old man with a history of COVID-19, re-presenting to the emergency department 4 days post-discharge with hypoxia and breathlessness. Note progressive consolidation and architectural distortion in the lower lobes occurring in the interval between the two films. (c,d) Axial unenhanced CT images of the chest in the same patient. Note peripheral consolidation with peri-lobular pattern (blue arrows) and bronchocentric ground-glass opacification with traction bronchiectasis (red arrows), architectural distortion (white arrow) with associated volume loss as seen by posterior retraction of the interlobar fissures (yellow arrows).

thromboembolic disease in COVID-19, which also conclude higher prevalence of peripherally located pulmonary emboli and lack of relationship between parenchymal disease and PTD. ${ }^{35-38}$ There are recent pathophysiological data $^{39}$ suggesting that an intravascular inflammatory process occurrs in COVID-19 leading to microangiopathic endothelial damage. Given that the majority of cases in the present cohort had segmental and subsegmental PTD, the present results also support the hypothesis that the vascular disease starts in the small vessels within the lungs. Virtually all cases developed the PTD in the second to third week of COVID-19 disease indicating that the thromboembolic disease process occurs later during the course of the disease. The present data suggest that the pulmonary thromboembolic event is common later in the course of the disease (second and third week of symptomatic disease), but it is currently unknown if thrombosis would also be encountered commonly if a CTPA was performed earlier.

The recommended UK-wide guidance at the time of our study was published by BSTI, which is based on expert consensus rather than real world data. We used the BSTI guidance for characterisation of the CT patterns and the extent of disease on the basis of our current clinical practice as opposed to the more recently introduced reporting systems such as CO-RADS. ${ }^{40}$ In the present cohort, better interobserver agreement was found using BSTI guidance in comparison with reported kappa values of 0.47 for CO-RADS system; however, the present results showed that interobserver agreement for the patterns of parenchymal abnormalities was at best moderate, indicating that BSTI guidance may require revision with more specified definitions for disease patterns. It should also be noted that the majority cases in the present cohort had moderate to severe disease, which could mask specific features of COVID-19 with peripheral and lower lobe predominant round ground glass opacities more visible in mild disease. This may also be the reason for a relatively large proportion of the present patients falling into the indeterminate category because of associated features of diffuse alveolar damage and fibrosis. A proportion of the present cohort also demonstrated features suggestive of fibrosis. Although the numbers are relatively small in the present cohort, given the number of patients affected globally, this finding indicates that a substantial number of patients with COVID-19 may progress to fibrosing interstitial lung disease, irrespective of the presence of ARDS.

A large proportion of patients with COVID-19 were found to have enlarged intrathoracic lymph nodes, which is in 
Table 4

The incidence of lymph nodes enlargement and pleural effusion among different CT patterns.

\begin{tabular}{|c|c|c|c|c|}
\hline & \multicolumn{4}{|c|}{ Consensus CT patterns } \\
\hline & Classic & Probable & Indeterminate & $\begin{array}{l}\text { Non- } \\
\text { COVID-19 }\end{array}$ \\
\hline $\begin{array}{l}\text { Total } \\
\text { Lymph node } \\
\text { enlargement }\end{array}$ & 20 & 24 & 40 & 9 \\
\hline No enlargement & $14(70 \%)$ & $16(66.7 \%)$ & $23(57.5 \%)$ & $6(66.7 \%)$ \\
\hline $\begin{array}{l}\text { Enlargement } \\
\text { Pleural effusion }\end{array}$ & $6(30 \%)$ & $8(33.3 \%)$ & 17 (42.5\%) & $3(33.3)$ \\
\hline $\begin{array}{l}\text { No pleural } \\
\text { effusion }\end{array}$ & $15(75 \%)$ & $24(100 \%)$ & $30(75 \%)$ & $4(44.4 \%)$ \\
\hline Pleural effusion & $5(25 \%)$ & $0(0 \%)$ & $10(25 \%)$ & $5(55.6 \%)$ \\
\hline
\end{tabular}

distinction to the initial report from China suggesting only $6 \%$ incidence of lymph node enlargement in hospitalised patients. ${ }^{41}$ Recent correspondence by French investigators ${ }^{42}$ showed up to $66 \%$ incidence of lymph node enlargement in COVID-19 patients admitted to intensive care unit. This indicates controversy surrounding the issue of lymph node enlargement, which could be partly due to differences in study cohorts. No co-existing pathogens were found that could explain lymph node enlargement in the majority of the cases, nor can the size of the lymph nodes be attributed to the presence of fibrosis. Similarly, pleural effusion is regarded as a non-COVID-19 feature ${ }^{12}$; however, this is also relatively common in the present cohort of confirmed COVID-19 cases, but may be a feature of comorbidities.

There are limitations to the present study. The sample size is relatively small, and the data were collected retrospectively comprising patients admitted to hospital, which result in selection bias of patients with more severe disease.

In conclusion, the high incidence of PTD in COVID-19 patients with severe disease undergoing CTPA, current lack of reliable risk stratification tools and symptoms of PTD overlapping with severe COVID-19 disease, raises the question of whether more CTPA studies should be performed in the second and third week of the illness. The present study suggests that patients with severe disease may require follow-up for long-term pulmonary complications.

\section{Conflict of interest}

The authors declare no conflict of interest.

\section{References}

1. Zhu N, Zhang D, Wang W, et al. A novel coronavirus from patients with pneumonia in China, 2019. N Engl J Med 2020;382:727-33.

2. European Centre for Disease Prevention and Control. COVID-19. Available at: https://www.ecdc.europa.eu/en/covid-19-pandemic [accessed May 3, 2020].

3. Guan W-J, Ni Z-Y, Hu Y, et al. Clinical characteristics of coronavirus disease 2019 in China. N Engl J Med 2020;382:1708-20.

4. Docherty AB, Harrison EM, Green CA, et al. Features of 16,749 hospitalised UK patients with COVID-19 using the ISARIC WHO Clinica
Characterisation Protocol: prospective observational cohort study. BMJ 2020;369:m1985.

5. Yang Y, Yang M, Shen C, et al. Evaluating the accuracy of different respiratory specimens in the laboratory diagnosis and monitoring the viral shedding of 2019-nCoV infections. medRxiv 2020. 2020.02.11.20021493.

6. Ai T, Yang Z, Hou H, et al. Correlation of chest CT and RT-PCR testing in coronavirus disease 2019 (COVID-19) in China: a report of 1014 cases. Radiology 2020:200642.

7. Revel MP, Parkar AP, Prosch H, et al. COVID-19 patients and the radiology department - advice from the European Society of Radiology (ESR) and the European Society of Thoracic Imaging (ESTI). Eur Radiol 2020:1-7.

8. Sverzellati N, Milone F, Balbi M. How imaging should properly be used in COVID-19 outbreak: an Italian experience. Diagn Interv Radiol 2020;26:204-6.

9. Raptis CA, Hammer MM, Short RG, et al. Chest CT and coronavirus disease (COVID-19): a critical review of the literature to date. AJR Am J Roentgenol 2020:1-4.

10. Sverzellati N, Milanese G, Milone F, et al. Integrated radiologic algorithm for COVID-19 pandemic. J Thorac Imaging 2020;35:228-33.

11. Bai HX, Hsieh B, Xiong Z, et al. Performance of radiologists in differentiating COVID-19 from viral pneumonia on chest CT. Radiology 2020:200823.

12. Johnstone A. Thoracic imaging in COVID-19. British Society Of Thoracic Imaging guidelines for the Reporting radiologist. Available at: https:// www.bsti.org.uk/standards-clinical-guidelines/clinical-guidelines/bsticovid-19-guidance-for-the-reporting-radiologist/ [accessed May 4, 2020].

13. ACR. Recommendations for the use of chest radiography and computed tomography (CT) for suspected COVID-19 infection. Available at: https:// www.acr.org/Advocacy-and-Economics/ACR-Position-Statements/ Recommendations-for-Chest-Radiography-and-CT-for-SuspectedCOVID19-Infection [accessed May 4, 2020].

14. Liang W, Guan W, Li C, et al. Clinical characteristics and outcomes of hospitalised patients with COVID-19 treated in Hubei (epicenter) and outside Hubei (non-epicenter): a nationwide analysis of China. Eur Respir J 2020;55:2000562.

15. Zhou F, Yu T, Du R, et al. Clinical course and risk factors for mortality of adult inpatients with COVID-19 in Wuhan, China: a retrospective cohort study. Lancet 2020;395:1054-62.

16. Desborough MJR, Doyle AJ, Griffiths A, et al. Image-proven thromboembolism in patients with severe COVID-19 in a tertiary critical care unit in the United Kingdom. Thromb Res 2020;193:1-4.

17. Fraissé M, Logre E, Pajot O, et al. Thrombotic and hemorrhagic events in critically ill COVID-19 patients: a French monocenter retrospective study. Crit Care 2020;24:275.

18. Tang N, Bai H, Chen X, et al. Anticoagulant treatment is associated with decreased mortality in severe coronavirus disease 2019 patients with coagulopathy. J Thromb Haemost 2020;18:1094-9.

19. Thachil J, Tang N, Gando S, et al. ISTH interim guidance on recognition and management of coagulopathy in COVID-19. I Thromb Haemost 2020; $18: 1023-6$.

20. Lippi G, Favaloro EJ. D-dimer is associated with severity of coronavirus disease 2019: a pooled analysis. Thromb Haemost 2020;18:1094-9.

21. Lippi G, Plebani M, Henry BM. Thrombocytopenia is associated with severe coronavirus disease 2019 (COVID-19) infections: a meta-analysis. Clin Chim Acta 2020;506:145-8.

22. Henry BM, de Oliveira MHS, Benoit S, et al. Hematologic, biochemical and immune biomarker abnormalities associated with severe illness and mortality in coronavirus disease 2019 (COVID-19): a meta-analysis. Clin Chem Lab Med 2020;58:1021-8. https://doi.org/10.1515/cclm-20200369 .

23. Leisman DE, Deutschman CS, Legrand M. Facing COVID-19 in the ICU: vascular dysfunction, thrombosis, and dysregulated inflammation. Intensive Care Med 2020;46:1105-8.

24. Leonard-Lorant I, Delabranche X, Severac F, et al. Acute pulmonary embolism in COVID-19 patients on CT angiography and relationship to D-dimer levels. Radiology 2020:201561.

25. Grillet F, Behr J, Calame P, et al. Acute pulmonary embolism associated with COVID-19 pneumonia detected by pulmonary CT angiography. Radiology 2020:201544. 
26. Oudkerk M, Büller HR, Kuijpers D, et al. Diagnosis, prevention, and treatment of thromboembolic complications in COVID-19: report of the National Institute for Public Health of the Netherlands. Radiology 2020:201629.

27. Wells PS, Anderson DR, Rodger M, et al. Derivation of a simple clinical model to categorize patients probability of pulmonary embolism: increasing the models utility with the SimpliRED D-dimer. Thromb Haemost 2000;83:416-20.

28. Robbie H, Wells AU, Jacob J, et al. Visual and automated CT measurements of lung volume loss in idiopathic pulmonary fibrosis. AJR Am J Roentgenol 2019;213:318-24.

29. Hansell DM, Bankier AA, MacMahon H, et al. Fleischner Society: glossary of terms for thoracic imaging. Radiology 2008;246:697-722.

30. Hansell D, Lynch D, McAdams HP, et al. Imaging of Diseases of the Chest. 5th edn. London: Elsevier; 2009.

31. Ascha M, Renapurkar RD, Tonelli AR. A review of imaging modalities in pulmonary hypertension. Ann Thorac Med 2017;12:61-73.

32. Ng CS, Wells AU, Padley SP. A CT sign of chronic pulmonary arterial hypertension: the ratio of main pulmonary artery to aortic diameter. $J$ Thorac Imaging 1999;14:270-8.

33. Cicchetti DV, Sparrow SA. Developing criteria for establishing interrater reliability of specific items: applications to assessment of adaptive behavior. Am J Ment Defic 1981;86:127-37.

34. Yan Z, Ip IK, Raja AS, et al. Yield of CT pulmonary angiography in the emergency department when providers override evidence-based clinical decision support. Radiology 2017;282:717-25.
35. Van Dam VF, Kroft LJM, Cannegieter SC, et al. Clinical and computed tomography characteristics of COVID-19 associated acute pulmonary embolism: a different phenotype of thrombotic disease? Thromb Res 2020;193:86-9.

36. Gervaise A, Bouzad C, Peroux E, et al. Acute pulmonary embolism in non-hospitalized COVID-19 patients referred to CTPA by emergency department. Eur Radiol 2020:1-8.

37. Poissy J, Goutay J, Caplan M, et al. Pulmonary embolism in COVID-19 patients: awareness of an increased prevalence. Circulation 2020, https://doi.org/10.1161/CIRCULATIONAHA.120.047430.

38. Bompard F, Monnier H, Saab I, et al. Pulmonary embolism in patients with COVID-19 pneumonia. Eur Respir J 2020:2001365.

39. Magro C, Mulvey JJ, Berlin D, et al. Complement associated microvascular injury and thrombosis in the pathogenesis of severe COVID-19 infection: a report of five cases. Transl Res 2020;220:1-13.

40. Prokop M, van Everdingen W, van Rees Vellinga T, et al. CO-RADS - a categorical CT assessment scheme for patients with suspected COVID19: definition and evaluation. Radiology 2020:201473.

41. Shi H, Han X, Jiang N, et al. Radiological findings from 81 patients with COVID-19 pneumonia in Wuhan, China: a descriptive study. Lancet Infect Dis 2020;20:425-34.

42. Valette X, du Cheyron D, Goursaud S. Mediastinal lymphadenopathy in patients with severe COVID-19. Lancet Infect Dis 2020;S1473-3099(20):30310-8. 